\title{
Cost-effectiveness analysis of camrelizumab in the second-line treatment for advanced or metastatic esophageal squamous cell carcinoma in China
}

\author{
Fan Yang ${ }^{1,2,3} \wedge$, Yu Fu ${ }^{1,2,3}$, Arun Kumar ${ }^{4}$, Mingsheng Chen $^{1,2,3}$, Lei Si ${ }^{5,6}$, Sirikan Rojanasarot ${ }^{7}$ \\ ${ }^{1}$ School of Health Policy \& Management, Nanjing Medical University, Nanjing, China; ${ }^{2}$ Creative Health Policy Research Group, Nanjing Medical \\ University, Nanjing, China; ${ }^{3}$ Center for Global Health, Nanjing Medical University, Nanjing, China; ${ }^{4}$ University of Minnesota College of Pharmacy, \\ Minneapolis, MN, USA; ${ }^{5}$ The George Institute for Global Health, UNSW Sydney, Kensington, NSW, Australia; ${ }^{6}$ UNSW Medicine, UNSW \\ Australia, Sydney, Australia; ${ }^{7}$ Boston Scientific, Marlborough, MA, USA \\ Contributions: (I) Conception and design: F Yang; (II) Administrative support: M Chen, L Si; (III) Provision of study materials or patients: Y Fu, \\ F Yang, A Kumar; (IV) Collection and assembly of data: Y Fu; (V) Data analysis and interpretation: F Yang, Y Fu, S Rojanasarot; (VI) Manuscript \\ writing: All authors; (VII) Final approval of manuscript: All authors. \\ Correspondence to: Dr. Fan Yang. School of Health Policy \& Management, Nanjing Medical University, 101 Longmian Avenue, Jiangning District, \\ Nanjing 211166, China. Email: yangfan512@njmu.edu.cn.
}

Background: This study aimed to evaluate the cost-effectiveness of camrelizumab versus chemotherapy as second-line treatment for patients with advanced/metastatic esophageal squamous cell carcinoma (ESCC) from the perspective of the Chinese healthcare system.

Methods: A trial-based Markov model was constructed using Excel to integrate clinical and economic data in a hypothetical cohort of advanced/metastatic ESCC patients with a 5-year time horizon. Clinical inputs were derived directly from the ESCORT trial (NCT03099382). Weibull distribution was used to fit transition probabilities extracted from the Kaplan-Meier curves. Cost inputs were estimated from the Beijing Medicine Sunshine Purchasing official website, local charges, publications and expert opinions. Deterministic and probabilistic sensitivity analyses were performed to test the robustness of the model results.

Results: At 5 years, camrelizumab had higher quality-adjusted life years (QALYs) (0.782 vs. 0.499) and higher cost (US\$31,537 vs. US\$6,998) than chemotherapy. The incremental cost-effectiveness ratio (ICER) was estimated to be US\$86,745 per QALY gained. The two primary parameters upon which this result was most sensitive were median overall survival of camrelizumab and camrelizumab cost. At a willingness-to-pay threshold of three times per capita gross domestic product (US\$30,094 per QALY gained), the probability of camrelizumab being cost-effective was $33.7 \%$.

Conclusions: Camrelizumab was not cost-effective as a second-line treatment for advanced/metastatic ESCC patients in China compared with chemotherapy.

Keywords: Advanced/metastatic esophageal squamous cell carcinoma (advanced/metastatic ESCC); costeffectiveness analysis; camrelizumab; second-line treatment

Submitted Apr 13, 2021. Accepted for publication Jun 22, 2021.

doi: 10.21037/atm-21-1803

View this article at: https://dx.doi.org/10.21037/atm-21-1803

\footnotetext{
$\wedge$ ORCID: 0000-0001-5075-4888.
} 


\section{Introduction}

Esophageal cancer is the sixth leading cause of death worldwide, accounting for more than half a million cancer deaths in 2018 (1). Of the two main histologic subtypes of esophageal cancer, esophageal squamous cell carcinoma (ESCC) is the predominant subtype in China (2). According to the Chinese National Central Cancer Registry, in 2015 ESCC was the fourth most diagnosed and fatal cancer in China (3). Prior work suggests that most patients with ESCC are not diagnosed at early stages and are therefore prone to metastasis, resistance, and recurrence, which contribute to its poor 5-year survival rate (4).

Due to diagnostic and therapeutic advancements in the management of ESCC, its age-standardized 5-year survival rate in China improved from 2003 to 2015 (5). However, the long-term survival of patients with ESCC remains low, and the disease still represents a substantial social and economic burden on the Chinese healthcare system (6). According to a prior disease burden study, the years of life lost (YLLs) from esophageal cancer in 2017 were 312 per 100,000 population. Additionally, the ranking of ESCC responsible for YLLs increased from $16^{\text {th }}$ in 1990 to $11^{\text {th }}$ in 2017 (7). In China, the average medical expenditure per esophageal cancer patient from 2002 to 2011 was approximately US\$5,471 and increased annually by $6.27 \%$ during that period (8).

The routine first-line treatment for patients with advanced or metastatic ESCC is chemotherapy (9), which continues until there is evidence of disease progression or intolerable toxicity. Single-agent chemotherapy such as docetaxel or paclitaxel is the standard second-line treatment $(9,10)$. However, currently available second-line chemotherapies for ESCC are associated with not only a poor relative 5 -year survival rate but also a significant rate of toxicity, including neurotoxicity, hematotoxicity, and gastrointestinal toxicity $(11,12)$. Immune checkpoint inhibitors have been proposed as an alternative second-line regimen for patients with advanced or metastatic ESCC. The ATTRACTION-3 and KEYNOTE-181 Phase III trials reported that the use of immunotherapy as secondline treatment resulted in improved overall survival and lower rates of toxicity than conventional chemotherapy $(13,14)$. Another Phase III trial, ESCORT, was the first randomized study in China that compared chemotherapy with camrelizumab, a programmed cell death protein-1 (PD-1) inhibitor, and found that patients treated with camrelizumab had improved overall survival and a better safety profile (15). Importantly, the proportion of grade 3 or worse treatment-related adverse reactions in this trial was lower in the camrelizumab cohort than chemotherapy (19\% vs. 40\%) (15). Despite the higher efficacy of the drug, the price of camrelizumab is much higher than the current standard of care, which may impose a substantial financial burden on the national healthcare system. This is particularly important consideration since camrelizumab was approved by China's National Medical Products Administration (NMPA) in 2020. It is therefore essential to perform an economic comparison of camrelizumab with conventional chemotherapy as a second-line therapy for advanced or metastatic ESCC to better understand the relative costs of the health outcomes of the two treatments.

The objective of our study was to assess the costeffectiveness of camrelizumab compared with routine chemotherapy as second-line management for advanced or metastatic ESCC patients from the perspective of the healthcare system in China.

We present the following article in accordance with the CHEERS reporting checklist (available at https://dx.doi. org/10.21037/atm-21-1803).

\section{Methods}

\section{Overview}

The cost-effectiveness of camrelizumab compared with chemotherapy as second-line therapy for advanced or metastatic ESCC in the Chinese population was assessed using an Excel-based (Microsoft, Redmond, WA, USA) Markov model (16). Clinical data were obtained from ESCORT (NCT03099382, March 2017), a multicentre, randomized, open-label, phase III study of camrelizumab (200 mg every 2 weeks) versus chemotherapy with docetaxel $\left(75 \mathrm{mg} / \mathrm{m}^{2}\right.$ every 3 weeks) or irinotecan $\left(180 \mathrm{mg} / \mathrm{m}^{2}\right.$ every 2 weeks) that was performed at 43 Chinese hospitals (15). We assumed that populated patients in the model had a median age of 60 , and that $80 \%$ of the patients had an Eastern Cooperative Oncology Group (ECOG) performance status equal to 1 or 0 . The model was constructed from the Chinese healthcare system perspective, with a lifetime horizon defined as 5 years. A 5 -year time horizon was selected because nearly $100 \%$ of patients in both study arms died by that timepoint according to a simulation of two 1,000-patient cohorts $(99.2 \%$ vs. $100.0 \%$ of camrelizumab and chemotherapy patients died at 5 years, respectively). In accordance with the China guidelines for 


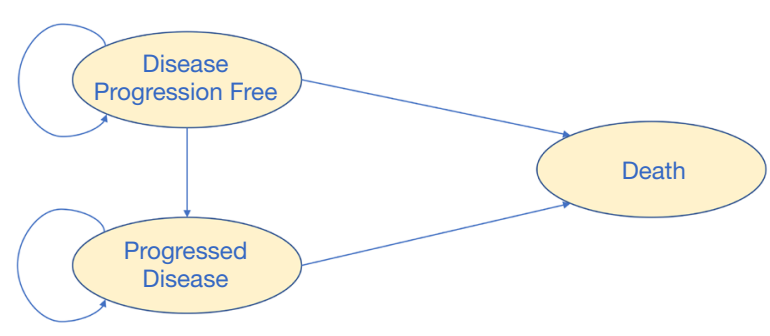

Figure 1 Model structure of camrelizumab and chemotherapy treatments as second-line therapy for advanced or metastatic esophageal squamous cell carcinoma.

pharmacoeconomic evaluations, costs and benefits were discounted at an annual rate of $5 \%$ (17).

The primary outcome of this analysis was the incremental cost-effectiveness ratio (ICER) of camrelizumab versus chemotherapy. ICER was defined as the difference in the cost of the two treatments divided by the difference in their quality-adjusted life years (QALYs), where QALY incorporated both length and quality of life into a single metric. Incremental cost per life year (LY) gained was also explored. The willingness-to-pay (WTP) threshold for study purposes was set at three-times the gross domestic product (GDP) per capita of China in 2019 (US\$30, 094/QALY).

\section{Patient patbway and model structure}

A hypothetical cohort of patients with a histologic or cytologic diagnosis of advanced or metastatic ESCC who had progressed on or were intolerant to first-line chemotherapy were randomly assigned to camrelizumab or a second-line chemotherapy. Camrelizumab $200 \mathrm{mg}$ was administered intravenously over 30 minutes on day 1 of each 14-day cycle. Patients with administered chemotherapy were given either docetaxel $75 \mathrm{mg} / \mathrm{m}^{2}$ on day 1 of each 21 -day cycle or irinotecan $180 \mathrm{mg} / \mathrm{m}^{2}$ on day 1 of each 14-day cycle at the discretion of the ESCORT trial's investigator. Treatments were continued until the disease progressed or intolerable toxicity was diagnosed. The Markov process of this analysis included three health states: progression-free survival (PFS), progressed disease (PD), and death. All simulated patients were assumed to start in the PFS state, and either stay in that same state or move to another state in the next cycle. After disease progression, post-discontinuation chemotherapy was adopted based on the ESCORT trial study protocol. The cycle length of the model was 21 days, which was used to adhere to the treatment regimens of the ESCORT trial. All model data were adjusted to this cycle length. Model structure is shown in Figure 1.

\section{Statistical analysis}

\section{Clinical data}

Clinical inputs, including the clinical efficacy of camrelizumab and chemotherapy, are presented in Table 1. The incidence of adverse events for this analysis was derived directly from the ESCORT trial. Adverse events included asthenia, anaemia, decreased white blood cell count, decreased appetite, diarrhea, decreased neutrophil count, nausea, and vomiting. As individual patient data (IPD) were not available, the GetData Graph Digitizer was used to derive time and survival probabilities by digitizing the Kaplan-Meier survival curves of PFS and overall survival (OS) from the trial. The best fitting distribution model was selected by the methods of both statistical tests (Akaike information criterion and Bayesian information criterion) and visual inspection. The Akaike information criterion and Bayesian information criterion were calculated using survival analyses with Stata (StataCorp LLC, College Station, TX, USA). The lowest values from both estimators were then selected (24). Akaike information criterion and Bayesian information criterion calculations for different distributions are presented in Table S1. The 2-parametric Weibull distribution model was chosen to fit the data extracted from the survival curves for PFS and OS (Table 1). Results of the survival curve simulation are presented in Figure 2. Transition probabilities were calculated using the following formula: $\mathrm{P}(\mathrm{t})=1-\exp \left[\lambda(\mathrm{t}-1)^{\gamma}-\lambda \mathrm{t}^{\gamma}\right]$ where $\lambda$ represented the scale of the distribution, $\gamma$ represented the shape of the distribution, and $t$ was the Markov cycle. The mortality rate of the general population in China in 2019 was used as the transition probability of PFS to death (23).

\section{Cost data}

Direct medical costs were calculated from the Chinese healthcare system perspective. This analysis included the costs of camrezulimab and chemotherapy, follow-up administration, post-discontinuation chemotherapy and treatment-related adverse events grade 3 or worse. The price of camrelizumab in China was US\$2,802 per $200 \mathrm{mg}$. The prices of routine chemotherapies and post-discontinuation chemotherapy were derived from the cost database available on the Beijing Medicine Sunshine Purchasing official 
Table 1 Key parameters for the Markov model

\begin{tabular}{|c|c|c|c|c|}
\hline Parameter & Value & Range & Distribution & Source \\
\hline Chemotherapy & 678 & $542-813$ & Gamma & Beijing Medicine Sunshine Purchasing Official Website (18) \\
\hline Camrelizumab & 4,203 & $970-4,203$ & Gamma & Local Charge \\
\hline Follow-up administration & 48 & $38-57$ & Gamma & Expert Opinion \\
\hline \multicolumn{5}{|c|}{ Grade 3 or worse treatment-related adverse events } \\
\hline Asthenia & 115 & $92-138$ & Gamma & Chongqing et al. (19) \\
\hline Anaemia & 531 & $425-638$ & Gamma & Wu et al. (20) \\
\hline Decreased white blood cell count & 466 & $373-559$ & Gamma & Gu et al. (21) \\
\hline Decreased neutrophil count & 354 & $283-425$ & Gamma & Expert Opinion \\
\hline Nausea & 71 & $57-85$ & Gamma & Expert Opinion \\
\hline Vomiting & 71 & $57-85$ & Gamma & Expert Opinion \\
\hline \multicolumn{5}{|l|}{ Utility } \\
\hline PFS & 0.74 & $0.59-0.89$ & Beta & Al-Batran et al. (22) \\
\hline PD & 0.56 & $0.45-0.67$ & Beta & Al-Batran et al. (22) \\
\hline \multicolumn{5}{|l|}{ Clinical data } \\
\hline \multicolumn{5}{|l|}{ Parameters of Weibull models } \\
\hline \multirow[t]{2}{*}{ Camrelizumab OS } & $\lambda=0.04$ & - & Fixed in PSA & The Weibull model \\
\hline & $\gamma=1.11$ & - & Fixed in PSA & The Weibull model \\
\hline \multirow[t]{2}{*}{ Chemotherapy OS } & $\lambda=0.04$ & - & Fixed in PSA & The Weibull model \\
\hline & $\gamma=1.28$ & - & Fixed in PSA & The Weibull model \\
\hline
\end{tabular}

Others

Discount rate

\begin{tabular}{lcccc} 
Cost & $5 \%$ & $0-8 \%$ & Fixed in PSA & China guidelines for pharmacoeconomic evaluations (17) \\
QALY & $5 \%$ & $0-8 \%$ & Fixed in PSA & China guidelines for pharmacoeconomic evaluations (17) \\
$\begin{array}{l}\text { Age-adjusted death rates in China } \\
\text { in } 2019\end{array}$ & $7.14 \%$ & $5.72-8.57 \%$ & Fixed in PSA & National Bureau of Statistics of China (23) \\
\hline
\end{tabular}

$\lambda$ represents the scale of the distribution of Weibull survival model; $\gamma$ represents the shape of the distribution of Weibull survival model. QALY, quality-adjusted life year; PFS, progression-free survival; PD, progressed disease; OS, overall survival; PSA, probabilistic sensitivity analysis. 

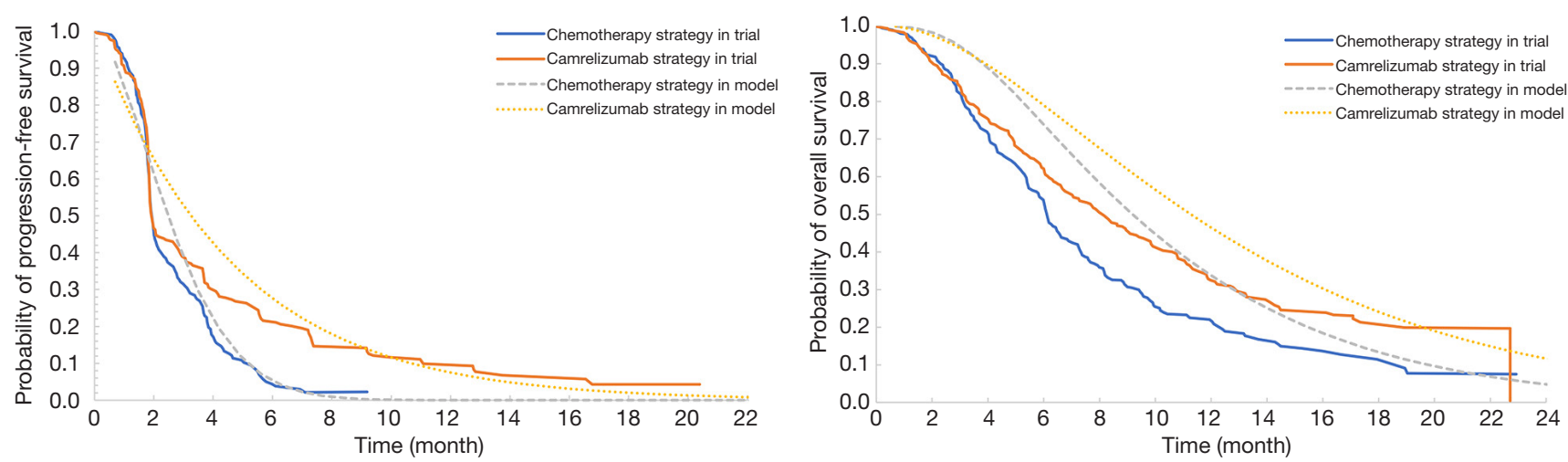

Figure 2 Estimated Weibull survival curves for progression-free survival and overall survival derived from the ESCORT trial.

website (18). A body surface area of $1.72 \mathrm{~m}^{2}$, with a typical weight of $65 \mathrm{~kg}$ and a height of $1.64 \mathrm{~m}$ were used to calculate chemotherapy drug doses (25). Follow-up administration components were derived from the guidelines of the Chinese Society of Clinical Oncology, including CT scans of the chest and abdomen, gastroscopy, ultrasound of the neck, and upper digestive tract radiography (26). Followup administration costs were then estimated by oncology experts. The costs of treating included treatment-related adverse events were derived from published literature or expert opinion (19-21). All costs of one cycle were inflated to match 2020 price and were presented in US dollars (Table 1).

\section{Health state utilities}

Health state utility (HSU) values were obtained from published literature and are shown in Table 1 . The estimated HSU values were 0.74 for PFS (22), 0.56 for PD (22), and 0 for death.

\section{Sensitivity analysis}

Sensitivity analyses were performed to investigate the robustness of the model. In the deterministic sensitivity analysis, key parameters were varied to assess their impact on ICER. As drug price negotiation occurs yearly in China, the cost of camrelizumab varied by $-76.92 \%$, meaning that the lowest price of camrelizumab per cycle was US $\$ 970$. This took into consideration the average discount of a branded drug based on the previously negotiated price on the National Reimbursement Drug List (NRDL) in China and the current Patient Assistance Program (PAP) cost of camrelizumab. Discount rates for both clinical and cost inputs varied from 0 to $8 \%$. Probabilistic sensitivity analysis was performed to assess the impact of uncertainty on model inputs using a Monte-Carlo simulation with 1,000 iterations. Results were presented as cost-effectiveness plane and cost-effectiveness acceptability curves. The ranges and parametric distributions of the included parameters are shown in Table 1.

\section{Ethical statement}

The study was conducted in accordance with the Declaration of Helsinki (as revised in 2013). This study was based on mathematical modeling and therefore no ethical approval was required by the ethics committee.

\section{Results}

\section{Base-case analysis results}

The base-case analysis over a lifetime horizon of 5 years showed that patients treated with camrelizumab had $1.35 \mathrm{LYs}$ and 0.782 QALYs at a total cost of US\$31,537. Patients treated with chemotherapy had 0.85 LYs and 0.499 QALYs at a total cost of US\$6,998. Camrelizumab therefore added an additional 0.50 LYs and 0.283 QALYs at an added cost of US\$24,539 compared with chemotherapy. The ICER was estimated to be US\$49,078 per LY gained and US\$86,745 per QALY gained (Table 2). Based on the ICER estimates, the camrelizumab treatment regimen was not cost-effective in China at a WTP threshold of three times per capita GDP (US\$30,094). Using the same WTP threshold, camrelizumab would be cost-effective compared with chemotherapy at a price of US\$1,032/200 mg. 
Table 2 Base-case life years, QALYs and costs of camrelizumab versus chemotherapy at a lifetime horizon of 5-years

\begin{tabular}{lccr}
\hline Parameter & Camrelizumab & Chemotherapy & Incremental \\
\hline LY & 1.35 & 0.85 & 0.50 \\
QALY & 0.782 & 0.499 & 0.283 \\
Cost & US\$31,537 & US $\$ 6,998$ & US $\$ 24,539$ \\
Cost per LY gained & - & - & US $\$ 49,078$ \\
Cost per QALY gained & - & - & US $\$ 86,745$ \\
\hline
\end{tabular}

LY, life year; QALY, quality-adjusted life year.

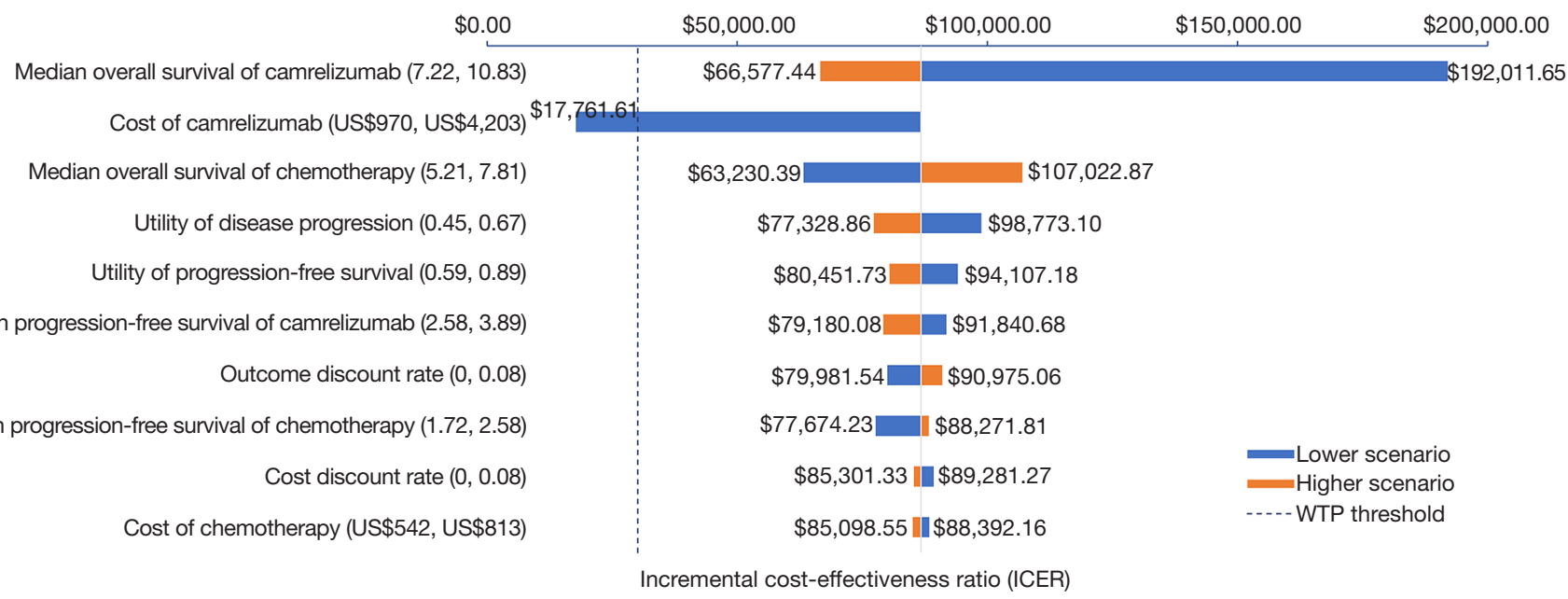

Figure 3 Deterministic sensitivity analysis tornado diagram of the top 10 most influential parameters.

\section{Sensitivity analysis results}

\section{Deterministic sensitivity analysis}

The top 10 parameters that most strongly influenced the deterministic sensitivity analysis are presented as a tornado diagram in Figure 3. An ICER of US\$86,745/QALY was used as the baseline values for the tornado diagram. The median OS of camrelizumab and the cost of camrelizumab were the most impactful parameters on the model results. The median OS of chemotherapy, the utility of PD, the utility of PFS, the median PFS of camrelizumab, the discount rate of outcome, and the median PFS of chemotherapy had a moderate impact on the model results. Changes in other parameters, such as the discount rate of cost and the cost of chemotherapy, had a minor influence on the robustness of the model's results.

\section{Probabilistic sensitivity analysis}

The results of the probabilistic sensitivity analysis are summarized as a scatterplot and a cost-effectiveness acceptability curve. As the WTP threshold was set at US\$30,094 per QALY gained, the probability of camrelizumab being cost-effective was only $33.7 \%$ (Figure 4). According to the cost-effectiveness acceptability curve (Figure 5), camrelizumab was not cost-effective compared with chemotherapy unless the WTP increased to at least US\$53,500.

\section{Discussion}

The present work was a trial-based cost-effectiveness analysis of camrelizumab versus chemotherapy as a second-line treatment of advanced or metastatic ESCC for Chinese patients utilizing clinical inputs from the ESCORT trial, a randomized, open-label, phase III study conducted at 43 hospitals in China. The results of this study showed that the ICER for camrelizumab versus chemotherapy was US\$86,745 per QALY gained, suggesting that camrelizumab was not a cost-effective 


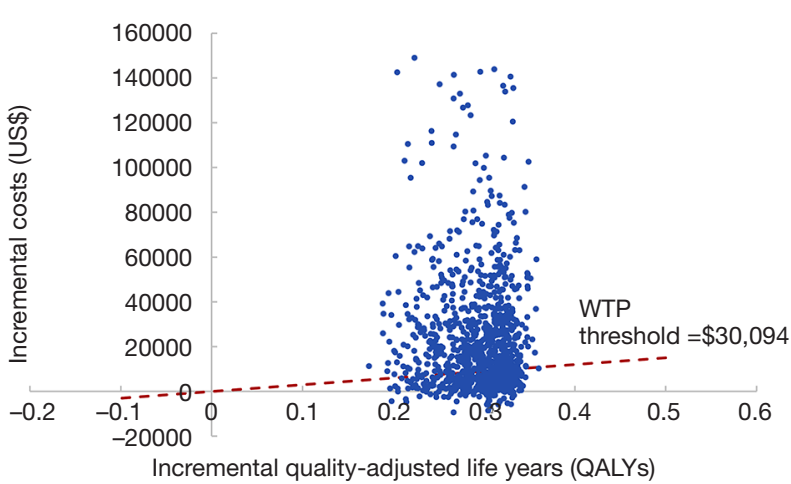

Figure 4 Scatterplot of the incremental cost-effectiveness ratio of camrelizumab $v s$. chemotherapy.

treatment option compared with routine chemotherapy from the Chinese healthcare system perspective. ESCC is the fourth most common cause of cancer-related death in the Chinese population, which used to have few secondline treatment options for patients with advanced or metastatic ESCC (27). The study findings of this work can provide Chinese decision makers with a price negotiation tool for camrelizumab, which is clinically useful but economically unreasonable at its current price.

This study is the first cost-effectiveness analysis of camrelizumab in the Chinese population, as few works have examined the economic impact of treatment options for ESCC. A majority of previously published studies on ESCC focused on diagnostic and therapeutic strategies, such as endoscopic screening, esophagectomy, neoadjuvant chemoradiotherapy followed by surgery or chemoradiation followed by esophagectomy (28-31). One study evaluated the cost-effectiveness of nivolumab, another PD-1 inhibitor that has been approved and recommended as treatment for advanced or metastatic ESCC in China. This multinational trial-based study from the Chinese society perspective found that nivolumab was not a cost-effective option as a second-line treatment for advanced or refractory ESCC compared with chemotherapy as the ICER was US\$136,709.35 per QALY gained (32). This finding is consistent with the base-case result of the present study where the ICER was US\$86,745 per QALY gained when comparing camrelizumab with chemotherapy. The prices of PD-1 inhibitors in China are substantially higher than those of conventional chemotherapy (US\$2,802/200 $\mathrm{mg}$ for camrelizumab and US $\$ 1,399 / 100 \mathrm{mg}$ for nivolumab (32) versus US\$678/cycle for chemotherapy). This study also found that the major parameters that influenced the model

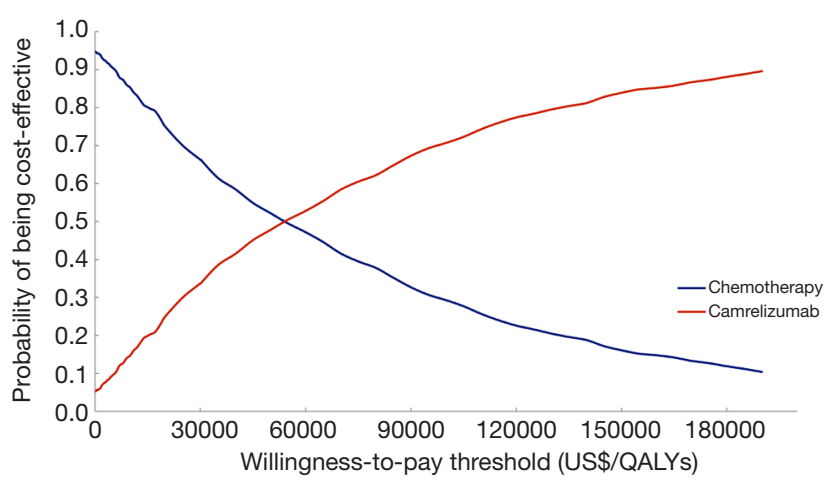

Figure 5 Cost-effectiveness acceptability curves.

results were the duration of PFS in nivolumab group and the cost of nivolumab, which were similar to the one-way sensitivity analysis findings of the present work. Compared with chemotherapy, camrelizumab is not cost-effective at the price of US $\$ 2,802 / 200 \mathrm{mg}$, but would become costeffective at the price of US\$1,032/200 mg. This study provides critical information to Chinese decision makers as they negotiate the price of camrelizumab. Lowering the price of this PD-1 inhibitor could help improve patient access to a treatment with a better safety profile and longer overall survival rates than conventional chemotherapies.

As cited in the 2019 China Guidelines for Pharmacoeconomic Evaluations (17), the threshold value of a QALY was set at three times the GDP per capita, which was US $\$ 30,094$. The probabilistic sensitivity analysis showed that at this threshold, the camrelizumab group was cost-effective in $33.7 \%$ of simulations. The ICER for the camrelizumab groups was greater than the thresholds recommended by richer developed countries, such as the $£ 20,000-30,000$ per QALY gained proposed by the United Kingdom National Institute for Health and Care Excellence (NICE) (33). If the prices of camrelizumab and chemotherapy are maintained, it is likely that camrelizumab will not be deemed cost-effective as second-line therapy for patients with advanced or metastatic ESCC in other countries as well.

The results of the ESCORT trial suggested that camrelizumab as a second-line therapy prolonged the overall survival of patients with advanced or metastatic ESCC compared with conventional chemotherapy as assigned by the physician (15). In the deterministic sensitivity analysis performed in this present study, the median OS of camrelizumab, had the highest impact on 
ICER. To obtain a lower ICER value, it is important for the physician to match the immunotherapy with suitable patients based on the likelihood of treatment efficacy. The findings of the ESCORT trial and the ATTRACTION-3 study also suggest that future studies are needed to confirm the association between PD-1 expression and patient benefit, and to identify other predictive biomarkers suggestive of the clinical utility of PD-1 inhibitors $(13,15)$.

From a policy standpoint, the findings of the present work suggest that the price of camrelizumab needs to be adjusted downward to reduce the financial burden on the healthcare system and to provide more value to Chinese patients. The deterministic sensitivity analysis revealed that the cost of camrelizumab was the second most influential parameter on ICER. Camrelizumab's manufacturer has a conditional patient assistant program that is designed to support a particular group of ESCC patients in China, which means there is the potential for a price reduction. In China, the yearly price negotiation period is the primary way for a novel drug to be included on the reimbursement list, and the 2019 negotiation resulted in an average fall in drug price of over $60 \%$ (34). There are more than 700 medications on the waiting list for addition to the national reimbursement drug list, which may lead to fierce competition for insurance access. Reduced drug pricing to minimize budgetary impact will be key elements considered by the government.

This study has several strengths. First, it is the first costeffectiveness analysis of camrelizumab versus standard chemotherapy in China, as well as the first economic evaluation of a PD-1 inhibitor for ESCC that utilizes a Chinese-based trial. The results of this analysis could therefore be taken into consideration by the National Healthcare Security Administration in the upcoming annual price negotiation of the NRDL. As this analysis was based on the Chinabased clinical trial, its findings are applicable to the Chinese healthcare system. This study also has some limitations. First, the utility of camrelizumab that was used in this study was derived from works that included European patients, and therefore may be different in the Chinese population. Second, camrelizumab was approved in China in June 2020 and has limited real-world data. Future economic evaluations should be performed using real-world evidence following the broader adoption of camrelizumab in the treatment of ESCC. Despite these limitations, this study can equip Chinese decision makers with improved health economic knowledge of camrelizumab as a potential second-line treatment regimen for advanced or metastatic ESCC in China.

\section{Conclusions}

A trial-based Markov model was constructed to integrate clinical and economic data in a hypothetical cohort of advanced or metastatic ESCC patients with a 5-year time horizon. This cost-effectiveness analysis indicated that the use of camrelizumab as a second-line treatment regimen for advanced or metastatic ESCC patients is not a cost-effective option compared with routine chemotherapy from the perspective of Chinese healthcare system.

\section{Acknowledgments}

Funding: This work was supported by the National Natural Science Foundation of China [Grant number: 71874086]; the China Medical Board [Grant number: 19-346]; and the Philosophy and Social Science Research Foundation for University of Jiangsu Province [Grant number: 2019SJA0295]. LS is supported by an NHMRC Early Career Fellowship [Grant number: GNT1139826].

\section{Footnote}

Reporting Checklist: The authors have completed the CHEERS reporting checklist. Available at https://dx.doi. org/10.21037/atm-21-1803

Data Sharing Statement: Available at https://dx.doi. org/10.21037/atm-21-1803

Conflicts of Interest: All authors have completed the ICMJE uniform disclosure form (available at https://dx.doi. org/10.21037/atm-21-1803). SR is Principal Health Economist at Boston Scientific. Boston Scientific has not involvement in this study. The content of this publication is under the sole responsibility of its author/publisher and does not represent the views or opinions of Boston Scientific Corporation. The other authors have no conflicts of interests to declare.

Ethical Statement: The authors are accountable for all aspects of the work in ensuring that questions related to the accuracy or integrity of any part of the work are appropriately investigated and resolved. The study was conducted in accordance with the Declaration of Helsinki (as revised in 2013. This study was based on mathematical modeling, therefore no ethical approval was required by the ethics committee. 
Open Access Statement: This is an Open Access article distributed in accordance with the Creative Commons Attribution-NonCommercial-NoDerivs 4.0 International License (CC BY-NC-ND 4.0), which permits the noncommercial replication and distribution of the article with the strict proviso that no changes or edits are made and the original work is properly cited (including links to both the formal publication through the relevant DOI and the license). See: https://creativecommons.org/licenses/by-nc-nd/4.0/.

\section{References}

1. Bray F, Ferlay J, Soerjomataram I, et al. Global cancer statistics 2018: GLOBOCAN estimates of incidence and mortality worldwide for 36 cancers in 185 countries. CA Cancer J Clin 2018;68:394-424.

2. Abnet CC, Arnold M, Wei WQ. Epidemiology of Esophageal Squamous Cell Carcinoma. Gastroenterology 2018;154:360-73.

3. Chen $\mathrm{W}$, Zheng R, Baade PD, et al. Cancer statistics in China, 2015. CA Cancer J Clin 2016;66:115-32.

4. Rustgi AK, El-Serag HB. Esophageal carcinoma. N Engl J Med 2014;371:2499-509.

5. Zeng $\mathrm{H}$, Chen $\mathrm{W}$, Zheng R, et al. Changing cancer survival in China during 2003-15: a pooled analysis of 17 population-based cancer registries. Lancet Glob Health 2018;6:e555-67.

6. Sun D, Cao M, Li H, et al. Cancer burden and trends in China: A review and comparison with Japan and South Korea. Chin J Cancer Res 2020;32:129-39.

7. Zhou M, Wang H, Zeng X, et al. Mortality, morbidity, and risk factors in China and its provinces, 1990-2017: a systematic analysis for the Global Burden of Disease Study 2017. Lancet 2019;394:1145-58.

8. Guo LW, Huang HY, Shi JF, et al. Medical expenditure for esophageal cancer in China: a 10-year multicenter retrospective survey (2002-2011). Chin J Cancer 2017;36:73.

9. Kitagawa Y, Uno T, Oyama T, et al. Esophageal cancer practice guidelines 2017 edited by the Japan esophageal society: part 2. Esophagus 2019;16:25-43.

10. Stahl M, Mariette C, Haustermans K, et al. Oesophageal cancer: ESMO Clinical Practice Guidelines for diagnosis, treatment and follow-up. Ann Oncol 2013;24 Suppl 6:vi51-6.

11. Muro K, Hamaguchi T, Ohtsu A, et al. A phase II study of single-agent docetaxel in patients with metastatic esophageal cancer. Ann Oncol 2004;15:955-9.
12. Jimenez P, Pathak A, Phan AT. The role of taxanes in the management of gastroesphageal cancer. J Gastrointest Oncol 2011;2:240-9.

13. Kato K, Cho BC, Takahashi M, et al. Nivolumab versus chemotherapy in patients with advanced oesophageal squamous cell carcinoma refractory or intolerant to previous chemotherapy (ATTRACTION-3): a multicentre, randomised, open-label, phase 3 trial. Lancet Oncol 2019;20:1506-17.

14. Kojima T, Muro K, Francois E, et al. Pembrolizumab versus chemotherapy as second-line therapy for advanced esophageal cancer: Phase III KEYNOTE-181 study. J Clin Oncol 2019;37:4010-10.

15. Huang J, Xu J, Chen Y, et al. Camrelizumab versus investigator's choice of chemotherapy as second-line therapy for advanced or metastatic oesophageal squamous cell carcinoma (ESCORT): a multicentre, randomised, open-label, phase 3 study. Lancet Oncol 2020;21:832-42.

16. Hollman C, Paulden M, Pechlivanoglou P, et al. A Comparison of Four Software Programs for Implementing Decision Analytic Cost-Effectiveness Models. Pharmacoeconomics 2017;35:817-30.

17. Liu GE, Hu SL, Wu JH, et al. China guidelines for pharmacoeconomic evaluations 2020 (Chinese-English Version). Beijing, China: China Market Press; 2020.

18. Integrated Management Platform of Beijing Medicine Sunshine Purchase (in Chinese). Beijing: Pharmaceutical Centralized Purchasing Service Center; c2021 [cited 2021 May 15]. Available online: https://yp.bjmbc.org.cn/view/ index/guide.html

19. Chongqing T, Liubao P, Xiaohui Z, et al. Cost-utility analysis of the newly recommended adjuvant chemotherapy for resectable gastric cancer patients in the 2011 Chinese National Comprehensive Cancer Network (NCCN) Clinical Practice Guidelines in Oncology: Gastric Cancer. Pharmacoeconomics 2014;32:235-43.

20. Wu B, Ye M, Chen H, et al. Costs of trastuzumab in combination with chemotherapy for HER2-positive advanced gastric or gastroesophageal junction cancer: an economic evaluation in the Chinese context. Clin Ther 2012;34:468-79.

21. Gu X, Zhang Q, Chu YB, et al. Cost-effectiveness of afatinib, gefitinib, erlotinib and pemetrexed-based chemotherapy as first-line treatments for advanced non-small cell lung cancer in China. Lung Cancer 2019;127:84-9.

22. Al-Batran SE, Van Cutsem E, Oh SC, et al. Quality-oflife and performance status results from the phase III 
RAINBOW study of ramucirumab plus paclitaxel versus placebo plus paclitaxel in patients with previously treated gastric or gastroesophageal junction adenocarcinoma. Ann Oncol 2016;27:673-9.

23. National Economy was Generally Stable in 2019 with Main Projected Targets for Development Achieved. Beijing: National Bureau of Statistics of China; c2020 [cited 2020 August 2020]. Available online: http://www.stats.gov.cn/ english/PressRelease/202001/t20200117_1723398.html

24. Mario C. William G, Roberto G. Gutierrez, et al. An introduction to survival analysis using stata. 3rd ed. Texas: Stata Press; 2010.

25. Wu B, Li T, Cai J, et al. Cost-effectiveness analysis of adjuvant chemotherapies in patients presenting with gastric cancer after D2 gastrectomy. BMC Cancer 2014;14:984.

26. Committee of Guidelines of Chinese society of clinical oncology. Guidelines of Chinese society of clinical oncology (CSCO)- esophageal cancer. (Chinese Version). Beijing, China: People's Medical Publishing House; 2020.

27. Lin $Y$, Totsuka $Y, H e Y$, et al. Epidemiology of esophageal cancer in Japan and China. J Epidemiol 2013;23:233-42.

28. Yang J, Wei WQ, Niu J, et al. Cost-benefit analysis of esophageal cancer endoscopic screening in high-risk areas of China. World J Gastroenterol 2012;18:2493-501.

Cite this article as: Yang F, Fu Y, Kumar A, Chen M, Si L, Rojanasarot S. Cost-effectiveness analysis of camrelizumab in the second-line treatment for advanced or metastatic esophageal squamous cell carcinoma in China. Ann Transl Med 2021;9(15):1226. doi: 10.21037/atm-21-1803
29. Liu CY, Lin CS, Shih CS, et al. Cost-Effectiveness of Minimally Invasive Esophagectomy for Esophageal Squamous Cell Carcinoma. World J Surg 2018;42:2522-9.

30. Zhan M, Zheng H, Yang Y, et al. Cost-effectiveness analysis of neoadjuvant chemoradiotherapy followed by surgery versus surgery alone for locally advanced esophageal squamous cell carcinoma based on the NEOCRTEC5010 trial. Radiother Oncol 2019;141:27-32.

31. Salcedo J, Suen SC, Bian SX. Cost-effectiveness of chemoradiation followed by esophagectomy versus chemoradiation alone in squamous cell carcinoma of the esophagus. Cancer Med 2020;9:440-6.

32. Zhang PF, Xie D, Li Q. Cost-effectiveness analysis of nivolumab in the second-line treatment for advanced esophageal squamous cell carcinoma. Future Oncol 2020;16:1189-98.

33. National Institute for Health and Care Excellence. Guide to the Methods of Technological Appraisal. NICE, London (2013).

34. Jinglin H. Comprehensively deepen the reform of the medical security system. Beijing: National Healthcare Security Administration, 2020 [cited 2021 Jul 4]. Available online: http://www.nhsa.gov.cn/art/2020/4/22/ art_14_3044.html 
Supplementary

Table S1 Akaike information criterion and Bayesian information criterion values at different data distributions

\begin{tabular}{lccccc}
\hline \multirow{2}{*}{ Distribution } & \multicolumn{3}{c}{ OS } & & PFS \\
\cline { 2 - 5 } \cline { 3 - 5 } Weibull & AIC & BIC & AIC & BIC \\
Exponential & $1,260.24$ & $1,272.64$ & $1,199.67$ & $1,211.99$ \\
\hline
\end{tabular}

AIC, Akaike information criterion; BIC, Bayesian information criterion; OS, overall survival; PFS, progression free survival. 